\title{
Observations of river solute concentrations during ice formation
}

Ella L. DeGrandpre, Michael D. DeGrandpre, Benjamin Colman and H. Maurice Valett, University of Montana, Missoula, Montana, USA

Supplementary information. The following tables contain the data used in this study.

Table S1: Laboratory study results. Mean and standard deviations $(\mathrm{n}=3)$ of metal solution concentrations $(\mu \mathrm{M})$ listed in order of increasing atomic mass. Ionic radii are also given $(\mathrm{pm}=$ picometer $)$. Concentration factors $\left(\mathrm{C}_{\text {water }}: \mathrm{C}_{\mathrm{ice}}\right)$ ratios are plotted in Figure 3.

\begin{tabular}{|c|c|c|c|}
\hline metal cations & ionic radius $(\mathrm{pm})$ & $\begin{array}{c}\text { concentration in water } \\
(\mu \mathrm{M})\end{array}$ & $\begin{array}{c}\text { concentration in ice } \\
(\mu \mathrm{M})\end{array}$ \\
\hline $\mathrm{Li}$ & 76 & $158.9 \pm 9.4$ & $24.0 \pm 1.4$ \\
\hline $\mathrm{Mg}$ & 72 & $55.7 \pm 3.7$ & $9.3 \pm 0.7$ \\
\hline $\mathrm{K}$ & 138 & $188.9 \pm 0.1$ & $18.0 \pm 2.5$ \\
\hline $\mathrm{Ca}$ & 99 & $82.7 \pm 7.5$ & $13.3 \pm 1.3$ \\
\hline $\mathrm{Co}$ & 75 & $42.5 \pm 3.2$ & $7.2 \pm 0.3$ \\
\hline $\mathrm{Cu}$ & 73 & $71.7 \pm 2.4$ & $12.5 \pm 0.3$ \\
\hline $\mathrm{Zn}$ & 74 & $69.6 \pm 3.4$ & $11.6 \pm 0.2$ \\
\hline $\mathrm{Sr}$ & 112 & $37.2 \pm 1.4$ & $5.5 \pm 0.1$ \\
\hline $\mathrm{Ba}$ & 135 & $52.5 \pm 2.0$ & $7.2 \pm 0.3$ \\
\hline
\end{tabular}

Table S2: Ion chromatography and titration (alkalinity) results for the initial river ice study, plotted in Figure 4. Uncertainty based on duplicate analyses of the same sample averaged $\pm 3.0 \%$.

\begin{tabular}{|c|c|c|c|}
\hline & $\begin{array}{c}\text { ionic radius } \\
(\mathrm{pm})\end{array}$ & $\begin{array}{c}\text { concentration in water } \\
(\mu \mathrm{M})\end{array}$ & $\begin{array}{c}\text { concentration in ice } \\
(\mu \mathrm{M})\end{array}$ \\
\hline $\mathrm{F}$ & 133 & 11.0 & 3.7 \\
\hline $\mathrm{Cl}$ & 184 & 95.1 & 18.6 \\
\hline $\mathrm{SO}_{4}$ & 258 & 363 & 27.2 \\
\hline Alkalinity & 156 & 2920 & 532 \\
\hline
\end{tabular}


Table S3: Solute concentrations during the December 2016 ice formation event listed in order of atomic mass. Concentrations are $\mu \mathrm{M}$. Data are plotted in Figure 5A. Uncertainty based on duplicate analyses of the same sample averaged $\pm 4.7 \%$.

\begin{tabular}{|c|c|c|c|c|c|}
\hline solute & $\begin{array}{c}\text { ionic } \\
\text { radius (pm) }\end{array}$ & Dec. 2 & Dec. 6 & Dec. 8 & Dec. 11 \\
\hline $\mathrm{Na}$ & 102 & 292 & 320 & 332 & 324 \\
\hline $\mathrm{Mg}$ & 72 & 461 & 497 & 521 & 529 \\
\hline $\mathrm{Si}$ & 40 & 184 & 190 & 202 & 193 \\
\hline $\mathrm{K}$ & 138 & 33.7 & 41.1 & 41.4 & 42.4 \\
\hline $\mathrm{Ca}$ & 99 & 943 & 1025 & 1077 & 1062 \\
\hline $\mathrm{Cu}$ & 73 & 0.025 & 0.039 & 0.027 & 0.035 \\
\hline $\mathrm{Zn}$ & 74 & 0.046 & 0.092 & 0.079 & 0.074 \\
\hline $\mathrm{Sr}$ & 112 & 1.56 & 1.71 & 1.80 & 1.71 \\
\hline
\end{tabular}

Table S4: Solute concentrations during the December 2017 ice formation event listed in order of atomic mass. Concentrations are $\mu \mathrm{M}$. Data are plotted in Figure 5B. Uncertainty based on duplicate analyses of the same sample averaged $\pm 2.0 \%$.

\begin{tabular}{|c|c|c|c|c|c|c|c|}
\hline solute & $\begin{array}{c}\text { Ionic } \\
\text { radius } \\
(\mathrm{pm})\end{array}$ & Dec. 9 & Dec. 10 & Dec. 11 & Dec. 12 & Dec. 13 & Dec. 15 \\
\hline $\mathrm{Na}$ & 102 & 267 & 285 & 290 & 309 & 291 & 301 \\
\hline $\mathrm{Mg}$ & 72 & 454 & 474 & 484 & 495 & 496 & 482 \\
\hline $\mathrm{Si}$ & 40 & 192 & 197 & 203 & 210 & 202 & 204 \\
\hline $\mathrm{K}$ & 138 & 41 & 44 & 44 & 49 & 46 & 46 \\
\hline $\mathrm{Ca}$ & 99 & 954 & 1001 & 1023 & 1063 & 1034 & 1030 \\
\hline $\mathrm{Sr}$ & 112 & 1.66 & 1.76 & 1.78 & 1.90 & 1.79 & 1.83 \\
\hline
\end{tabular}

